Documents pour l'histoire du français langue étrangère ou seconde

$24 \mid 1999$

Les auteurs classiques français dans l'enseignement du F.L.E. (18e et 19 e siècles)

\title{
Les Caractères de Jean de La Bruyère
}

\section{Christian Pelletier}

\section{(2) OpenEdition \\ 1 Journals}

\section{Édition électronique}

URL : https://journals.openedition.org/dhfles/3023

DOI : 10.4000/dhfles.3023

ISSN : 2221-4038

Éditeur

Société Internationale pour l'Histoire du Français Langue Étrangère ou Seconde

\section{Édition imprimée}

Date de publication : 1 décembre 1999

Pagination : p.201-204

ISSN : 0992-7654

\section{Référence électronique}

Christian Pelletier, "Les Caractères de Jean de La Bruyère ", Documents pour I'histoire du français langue étrangère ou seconde [En ligne], 24 | 1999, mis en ligne le 04 février 2015, consulté le 27 mai 2021. URL : http://journals.openedition.org/dhfles/3023 ; DOI : https://doi.org/10.4000/dhfles.3023

Ce document a été généré automatiquement le 27 mai 2021.

(C) SIHFLES 


\title{
Les Caractères de Jean de La Bruyère
}

\author{
Christian Pelletier
}

1 Les Caractères ou les mœurs de ce siècle constituent dans la littérature française classique une œuvre exceptionnelle à plusieurs titres. CEuvre unique par sa facture, à la fois très composite et très cohérente, elle est aussi l'unique œuvre originale de son auteur et lui assure au demeurant sa postérité.

2 Ce fut une œuvre au succès immédiat dès sa première édition au début de 1688 . Seize sections qui demeureront, Des ouvrages de l'esprit qui mènent à Des esprits forts (à noter que la dixième s'intitule originellement $\mathrm{Du}$ souverain et non $\mathrm{Du}$ Souverain ou de la République). Huit éditions du vivant de l'auteur en sept années (1688-1694), régulièrement augmentées à partir de la quatrième car si la première mouture comporte 420 remarques, maximes, portraits, formes brèves, adages, centons, formes gnomiques la huitième en compte 1 120. Les Caractères ont donc presque triplé de taille en atteignant l'âge de raison.

3 Publié anonymement grâce à un Privilège du Roy du 8 octobre 1687 accordé à Estienne Michallet, «Imprimeur du Roy, \& Marchand Libraire à Paris» l'ouvrage connaîtra de nombreuses et fameuses éditions au long des siècles.

4 Et des éditions très européennes. En 1688 édition à Lyon chez Thomas Amaulry, en 1693 à Bruxelles par Jean Léonard et la neuvième édition, posthume, en 1696 de nouveau par Estienne Michallet.

5 Signalons encore l'édition de 1726 à Amsterdam chez David Mortimer, celle de 1731 toujours à Amsterdam chez l'éditeur Changuion. En 1825 dans la Collection des moralistes français deux volumes chez l'éditeur Durey de Blois, le texte de La Bruyère étant précédé d'une notice de Suard sur l'auteur. En 1864 chez Morizot Paris. La liste serait très longue.

6 Dès 1699 (trois ans seulement après l'édition définitive) une traduction en anglais parait à Londres chez Bullord: «The Characters and the Manners of the age by monsieur de La Bruyère translated from the Greek and a prefatory discourse to them, by monsieur de La Bruyère to which is added a key to his cha-racters» («a key», on a une 
conception pragmatique de la littérature outre-Manche!), les deux parties en un volume in-octavo.

7 Une brève préface prétend résumer le projet de La Bruyère:

His design was to make his Reader a Wife, a Good Man, and a fine Gentleman, and his manner of prosecuting it is entirely new: no author on his subject has come up to his Force and Delicacy, nor has any so agreeably varied his style. [Son dessein fut de faire de son Lecteur une Femme, un Homme de Bien et un parfait Gentilhomme, et la façon dont il procède est entièrement nouvelle: nul auteur sur ce sujet n'a atteint cette Force et cette Délicatesse, ni varié aussi agréablement son style.] (C'est nous qui traduisons).

Indépendamment des difficultés de traduction d'un prosateur tel que La Bruyère un rapide sondage comparatiste peut apparaître éclairant. Considérons le portrait justement célèbre d'Onuphre qui rehausse encore l'œuvre à partir de 1691 et de sa sixième édition. Les éditions scolaires françaises ont le tort de vouloir résumer la complexité des portraits d'un mot. Gnathon, le gourmand, Lise, la coquette, Onuphre, l'hypocrite. Il s'agit d'une hypocrisie liée à une situation sociale et religieuse précise puisqu'elle est fausse dévotion et prend place dans la section De la mode.

Ce portrait est aussi un anti-Tartuffe. Quelque vingt années auparavant après les avatars que l'on sait Molière avait enfin pu faire jouer la pièce avec grand succès. Mais pour La Bruyère le portrait de Molière n'est qu'un «vrai faux» au sens où il n'est ni plausible ni crédible. Pour La Bruyère le faux dévot n'est pas tonitruant et spectaculaire, il est onctueux et discret. Il ne prend pas de risque en faisant des avances à la femme de son protecteur et ami:

Il sait où se trouvent des femmes plus sociables et plus dociles que celle de son ami, il ne les abandonne pas pour longtemps, quand ce ne serait que pour faire dire de soi dans le public qu'il fait des retraites; qui en effet pourrait en douter quand on le revoit paraître avec le visage exténué, et d'un homme qui ne se ménage point ? [[...] unless it be to give occasion to a public report that he retires from the world; and how indeed should they doubt it, when they fee his face faln away, as if he never indulged himself in the least.]

10 La traduction anglaise de 1699 ne conserve pas la marque stylistique de l'interrogation, contrairement à la traduction espagnole de 1947 publiée à Mexico et due à Luis R. Cuellar:

Sabe en dónde se encuentran mujeres más sociables y dóciles que la de su amigo y no las deja por largo tiempo, aun cuando esto solo sea para persuadir al público de que suele retirarse; y en efecto, ¿quién pudiera dudarlo al verlo con el semblante macilento de un hombre descuidado de sí mismo?.

$11 \mathrm{Au}$ cours du $19^{\mathrm{e}}$ siècle La Bruyère est à l'honneur. La Nouvelle Bibliothèque française des aspirants au baccalauréat ès lettres, publiée par M. Emile Lefranc avec la collaboration de plusieurs membres de l'Université lui consacre un volume. Il s'agit en fait du seul chapitre initial «Des ouvrages de l'esprit» annoté par A. Mottet. La collection ne comprend qu'une seule pièce de Molière (Le Misanthrope), Corneille, Pascal et Voltaire. La Fontaine est représenté par Philémon et Baucis et les Livres I à XII des Fables, Bossuet par deux oraisons funèbres, Massillon par les cinq premiers sermons du Petit Carême et un sermon sur la Mort.

12 Le volume consacré à La Bruyère contient un florilège des jugements portés sur Les Caractères qui sont autant de louanges apologétiques. Il est rappelé que La Harpe loue le moraliste (qu'il considère comme supérieur à La Rochefoucauld), Vauvenargues privilégie le style et que Chateaubriand s'exclame: «C'est un grand triomphe pour la 
religion que de compter parmi ses philosophes un Pascal et un La Bruyère». Enfin Walckenaer glorifie pour les potaches de 1849 le projet de l'auteur de «faire mieux ressortir la beauté de la vertu par le contraste des difformités du vice».

A la même époque paraît un Nouveau Manuel complet et méthodique des aspirants au baccalauréat (toujours le brevet français de bourgeoisie) qui en sa $21^{\mathrm{e}}$ édition comporte 1300 pages et 200 figures intercalées. Le projet pédagogique majeur consiste à apprendre le grec et le latin à l'aide d'une traduction intralittérale (le mot à mot tel qu'il est demandé à l'examen). Peu de notes en bas de page mais les auteurs du manuel n'hésitent pas à critiquer parfois les classiques en précisant par exemple «Cette phrase est lourde et embarrassée» ou bien en critiquant un jugement que La Bruyère avait formulé à propos de Rabelais en doutant de sa valeur littéraire.

14 Pour en finir sur une note d'humour et faire une rapide incursion dans notre siècle remarquons pour en venir au portrait d'Onuphre que les fameux Classiques Larousse sous couverture violette qui furent en situation de quasi monopole auprès des écoliers durant trois décennies (des années trente à soixante) expurgent le texte des lignes relatives aux incartades du faux dévot se laissant mener nous l'avons vu par les sortilèges de la chair plutôt que par les béatitudes de la chaire.

Sans signalement typographique d'une coupe qui passe ainsi inaperçue et constitue ainsi un exemple édifiant de tartufferie. Quant aux Nouveaux classiques Larousse actuellement en vigueur ils omettent purement et simplement ce portrait. Mais il est vrai, vous en conviendrez, que l'hypocrisie, pour parodier Molière, n'est pas un vice à la mode.

\section{AUTEUR}

\section{CHRISTIAN PELLETIER}

Université d'Angers 